\title{
Gas adsorption and capillary condensation in nanoporous alumina films
}

\author{
Fèlix Casanova ${ }^{1}$, Casey $\mathbf{E}$ Chiang $^{1}$, Chang-Peng $\mathbf{L i}^{1,2}$, \\ Igor V Roshchin ${ }^{1,3}$, Anne M Ruminski ${ }^{4}$, Michael J Sailor ${ }^{4}$ and \\ Ivan K Schuller ${ }^{1}$ \\ ${ }^{1}$ Physics Department, University of California-San Diego, La Jolla, CA 92093, USA \\ 2 Physics Department, University of Michigan-Ann Arbor, Ann Arbor, MI 48109, USA \\ ${ }^{3}$ Physics Department, Texas A\&M University, College Station, TX 77843, USA \\ ${ }^{4}$ Department of Chemistry and Biochemistry, University of California-San Diego, \\ La Jolla, CA 92093, USA \\ E-mail: casanova@physics.ucsd.edu
}

Received 14 February 2008, in final form 16 April 2008

Published 24 June 2008

Online at stacks.iop.org/Nano/19/315709

\begin{abstract}
Gas adsorption and capillary condensation of organic vapors are studied by optical interferometry, using anodized nanoporous alumina films with controlled geometry (cylindrical pores with diameters in the range of 10-60 nm). The optical response of the film is optimized with respect to the geometric parameters of the pores, for potential performance as a gas sensor device. The average thickness of the adsorbed film at low relative pressures is not affected by the pore size. Capillary evaporation of the liquid from the nanopores occurs at the liquid-vapor equilibrium described by the classical Kelvin equation with a hemispherical meniscus. Due to the almost complete wetting, we can quantitatively describe the condensation for isopropanol using the Cohan model with a cylindrical meniscus in the Kelvin equation. This model describes the observed hysteresis and allows us to use the adsorption branch of the isotherm to calculate the pore size distribution of the sample in good agreement with independent structural measurements. The condensation for toluene lacks reproducibility due to incomplete surface wetting. This exemplifies the relevant role of the fluid-solid (van der Waals) interactions in the hysteretic behavior of capillary condensation.
\end{abstract}

(Some figures in this article are in colour only in the electronic version)

\section{Introduction}

Nanoporous materials have promising properties for applications such as biosensing [1], chemical sensing [2] or nanotemplates [3], among others. These materials are also important in fundamental studies, such as phase transitions in confined geometries [4], because the relevant sizes are comparable to characteristic physical sizes which control transition mechanisms. In particular, capillary condensation can be studied in nanoporous materials with pore sizes somewhat larger than the adsorbate molecular diameter.

Capillary condensation during gas adsorption in nanopores has been largely studied in a wide variety of materials, comprised mostly of disordered/interconnected porous materials such as compacted powder, oxide xerogels or Vycor glass $[5,6]$, but direct comparison with theoretical work has been difficult due to the lack of ideal pore geometries [7, 8]. An example of this difficulty is the hysteresis in capillary condensation, a phenomenon which is still poorly understood $[6,9]$, unless an ideal system is used [10,11]. A complete understanding of basic mechanisms in gas adsorption and capillary condensation is essential for any future developments in vapor sensing using porous materials.

Recently, capillary condensation has been observed in anodized nanoporous alumina [9, 12], a suitable material which can be tailored by controlling the anodization parameters $[3,13,14]$. The nearly ideal cylindrical and not interconnected pore geometry has allowed us to validate the predictions of the classical Kelvin equation [5] for capillary condensation in pores with diameters $\geqslant 10 \mathrm{~nm}$, as we have shown in a previous work [12]. Experimental validation of the Kelvin equation in an ideal geometry was previously 


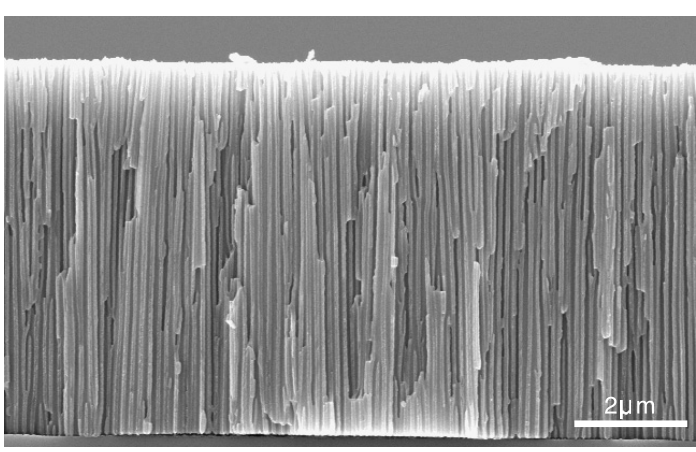

Figure 1. Cross-section SEM image of sample with a pore diameter of $61 \pm 12 \mathrm{~nm}$.

limited to systems consisting of space between mica surfaces (single slit-pores) $[15,16]$ and to pores $\leqslant 10 \mathrm{~nm}$ in diameter in molecular sieves [17]. Using optical interferometry to obtain adsorption-desorption isotherms, we showed that capillary evaporation of the liquid from the nanopores occurred at the equilibrium transition, i.e., at relative pressures given by the Kelvin equation, whereas capillary condensation occurred from metastable vapor states, giving rise to hysteresis [12]. This work is a follow-up study of gas adsorption and capillary condensation of organic vapors in nanoporous alumina films with non-interconnected cylindrical pores open at one end and well-controlled sizes in the $10-60 \mathrm{~nm}$ diameter range. From an applied point of view, to help optimization of gas sensor devices, we determine the dependence of the optical response of the film on the geometric parameters of the pores. From a basic point of view, we observe that the average thickness of the adsorbed film is unaffected by the confinement in the pores. Also, we are able to quantitatively describe the condensation for isopropanol with the Cohan model [5, 18], whereas the condensation for toluene does not show a reproducible path. This difference in behavior stresses the relevance of fluid-solid interactions in the description of the metastable states which cause hysteresis in capillary condensation.

\section{Experimental details}

Nanoporous alumina was fabricated from aluminum films evaporated on n-type Si using a two-step anodization $[3,13]$. The periodicity and diameters of the pores were controlled by the anodization parameters. A detailed study of anodized nanoporous alumina fabrication can be found in [3]. A set of 10 samples with the same nominal thickness $(\sim 6.15 \mu \mathrm{m})$ and different pore diameters $(10 \pm 2,12 \pm 2,13 \pm 3,18 \pm 4$, $22 \pm 5,27 \pm 3,33 \pm 7,43 \pm 10,48 \pm 10$ and $61 \pm 12 \mathrm{~nm})$ was prepared. Cross-sectional scanning electron microscopy (SEM) images (figure 1) showed that the pores are cylindrical, open at one end, propagate through the entire thickness of alumina, and are not connected to each other. Top view SEM images were obtained to determine the pore size distributions of the samples (figure 2). The porosities $\rho$ (fraction of empty volume) were obtained from the same images, yielding values from $\sim 0.1$ to 0.7 . The thicknesses $(L)$ of the porous films were measured with a profilometer, all yielding similar values

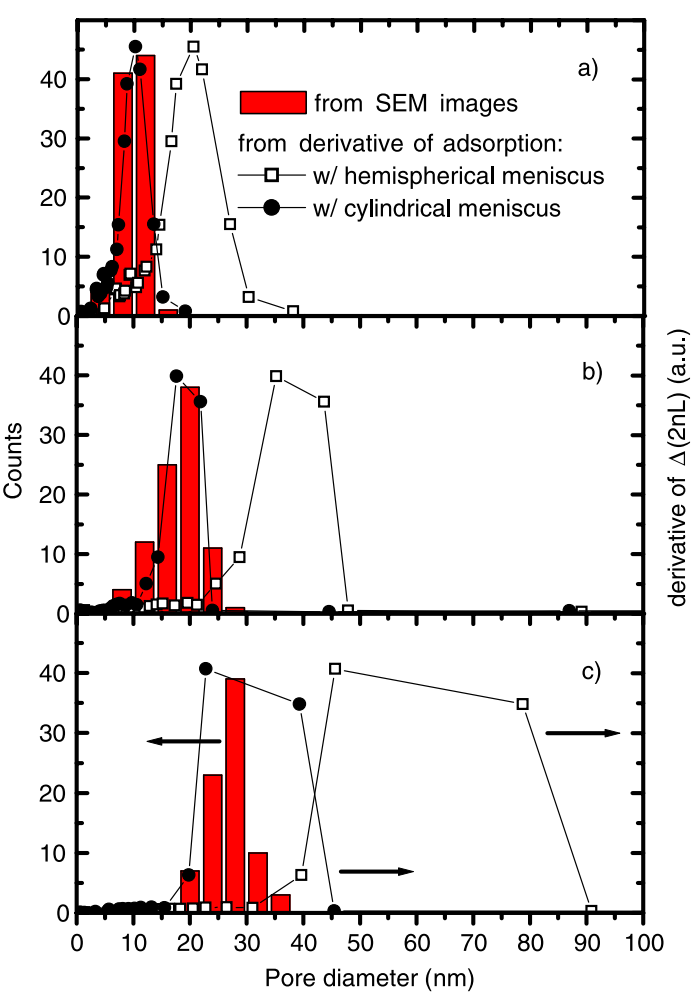

Figure 2. Pore diameter distribution for the samples with (a) $10 \pm 2 \mathrm{~nm}$, (b) $18 \pm 4 \mathrm{~nm}$ and (c) $27 \pm 3 \mathrm{~nm}$, as obtained from top view SEM images (histogram, left axis) and from the derivative of the adsorption isotherm for isopropanol combined with the Kelvin equation for hemispherical meniscus (empty squares, right axis) and with the Kelvin equation for cylindrical meniscus (solid circles, right axis).

$(\sim 6.15 \mu \mathrm{m})$. A second set of 4 samples with similar pore diameters $(12 \pm 2 \mathrm{~nm})$ and porosity $(\sim 0.45)$ but different thicknesses $(\sim 2.0,3.7,5.2$ and $6.5 \mu \mathrm{m})$ was also prepared and characterized.

Reflectance spectra were acquired using a tungsten white light source and a 350-1000 nm CCD spectrometer in a $90^{\circ}$ backscattering configuration, as previously described [1, 12]. The spectra display a series of fringes arising from the interference of the light reflected at the top and bottom interfaces of the porous alumina film. Since the pore diameters in the film are much smaller than the light wavelength $\lambda$, the film behaves as a single medium with an average refractive index, $n$. The wavelengths corresponding to the fringe maxima are given by the relationship $m \lambda=2 n L$, where $m$ is an integer. The term $2 n L$ is the effective optical thickness of the porous film. If the pores are filled with an analyte, $n$ of the film changes, causing a shift in the interference fringes. The value of $2 n L$ is obtained directly as the position of the peak in the fast Fourier transform of the reflectance spectrum expressed as a function of $1 / \lambda$. This value can be resolved to $\sim 1 \mathrm{~nm}[1,12]$.

Each sample was placed in a Teflon cell fitted with a glass window. The gas dosing was done with isopropanol or toluene vapors at a controlled temperature, while varying relative vapor pressures, $P / P_{\mathrm{s}}$, where $P_{\mathrm{s}}$ is the saturation vapor pressure. Ultra high purity (99.9999\% vol.) $\quad \mathrm{N}_{2}$ was used as a carrier gas. All analytes were of analytical 


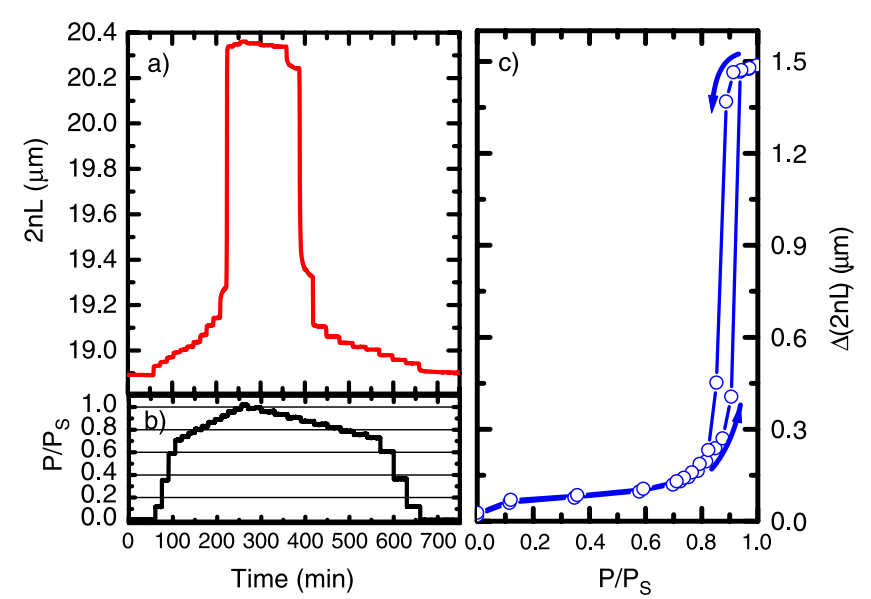

Figure 3. (a) Effective optical thickness, $2 n L$, measured as a function of time for a sample with pores of $18 \pm 4 \mathrm{~nm}$ in diameter while changing the relative pressure, $P / P_{\mathrm{s}}$, of isopropanol.

(b) Corresponding $P / P_{\mathrm{s}}$ of isopropanol as a function of time, which was first increased and then decreased in discrete steps. (c) Change in $2 n L$ as a function of $P / P_{\mathrm{s}}$ of isopropanol, obtained from the combined data in (a) and (b), which corresponds to an adsorption-desorption isotherm.

purity (99.9\% for isopropanol and $99.5 \%$ for toluene) and dried over $4 \AA$ molecular sieves. Saturated vapor analyte was generated by bubbling $\mathrm{N}_{2}$ through the liquid analyte at $25^{\circ} \mathrm{C}$ and then diluted by mixing the effluent with $\mathrm{N}_{2}$ using computer-controlled mass flow controllers. Total gas flow was kept constant at 500 standard cubic centimeters per minute (sccm). $P / P_{\mathrm{S}}$ were defined using the dilution ratio of the two mass flow controllers. Before any dosing was performed, the flow system, including the sample cell, was flushed with the ultra high purity $\mathrm{N}_{2}$. Simultaneously, the value of $2 n L$ of the sample was monitored. This value, very sensitive to any film adsorbed on the sample surface, first decreased with time, which indicated that the pore walls were being degassed. In less than $1 \mathrm{~h}$, when the system was fully degassed, $2 n L$ became constant.

To obtain the adsorption-desorption isotherms, the following procedure was used. The value of $2 n L$ was monitored (figure 3(a)) while $P / P_{\mathrm{s}}$ was increased and subsequently decreased stepwise (figure 3(b)). Following every change in $P / P_{\mathrm{s}}$, the system was allowed to reach equilibrium, so as to exclude transitional processes from consideration. For each step, the change in $2 n L[\Delta(2 n L)]$ with respect to the baseline obtained with empty pores $\left(P / P_{\mathrm{s}}=0\right)$ was plotted as a function of $P / P_{\mathrm{s}}$ (figure 3(c)). This plot provides the adsorption-desorption isotherm, because $\Delta(2 n L)$ is directly related to the volume of analyte adsorbed into the pores. Every isotherm was repeated at least three times to check reproducibility. It is worth noting that this optical interferometric technique has been used previously only to detect low gas concentrations in vapor sensors [2], but not full adsorption isotherms. This technique is different from other interferometric techniques that use multiple beam interference and a surface force apparatus to obtain adsorption isotherms $[10,11]$.

\section{Results and discussion}

The adsorption isotherms, for all samples and the two different analytes used, are of type IV, see for example figure 3(c). The sharp slope increase and the hysteresis (type H1) are associated with capillary condensation and evaporation within the nanopores. The isotherm levels off at high $P / P_{\mathrm{s}}$, when the pores become completely filled with liquid $[5,19]$.

To verify complete filling of pores at high $P / P_{\mathrm{s}}$, we measured $2 n L$ before (empty pores) and after immersion of the samples in liquid isopropanol and toluene. The obtained $\Delta(2 n L)$ values match the saturated values of the isotherms at high $P / P_{\mathrm{s}}, \Delta(2 n L)_{\text {sat }} .2 n L$ for empty and liquid-filled pores is also used to calculate $L$ and $\rho$ for each sample, using the Bruggeman effective medium approximation $[1,20]$ :

$$
(1-\rho)\left(\frac{n_{\mathrm{AlO}_{x}}^{2}-n^{2}}{n_{\mathrm{AlO}_{x}}^{2}+2 n^{2}}\right)+\rho\left(\frac{n_{\mathrm{p}}^{2}-n^{2}}{n_{\mathrm{p}}^{2}+2 n^{2}}\right)=0,
$$

where $n_{\mathrm{AlO}_{x}}=1.65$ is the refractive index of anodized alumina [21] and $n_{\mathrm{p}}$ is either 1 for empty pores or the refractive index of the corresponding liquid $n_{\mathrm{L}}$ for liquid-filled pores. The values of $L$ and $\rho$ obtained in these measurements are consistent with those previously obtained from profilometry and SEM image analyses.

Although the Bruggeman approximation is more accurate, we can also consider a simple-weighted average for the calculation of the refractive index,

$$
n \approx(1-\rho) n_{\mathrm{AlO}_{x}}+\rho n_{\mathrm{p}}
$$

This simplified approximation does not deviate substantially from equation (1) for the parameter range that we use, and allows us to visualize more easily the role of the sample geometry. From equation (2) we can derive a simple relationship for the value of $\Delta(2 n L)$,

$$
\Delta(2 n L) \approx 2 L \rho\left(n_{\mathrm{L}}-1\right) \alpha,
$$

where $\alpha$ is the ratio of the liquid-filled volume to the total volume of the pore: $\alpha=V_{\text {liquid }} / V_{\text {total }}$. At saturation, when $\alpha=1$, equation (3) implies that $\Delta(2 n L)_{\text {sat }}$ changes linearly with the thickness $L$ and the porosity $\rho$. Indeed, this behavior is observed in our samples as shown in figures 4(a) and (b) for $\Delta(2 n L)_{\text {sat }}$ as a function of $\rho$ and $L$, for different analytes. In completely filled pores, the optical signal can be increased by maximizing the porosity and the thickness of the nanoporous alumina films.

To understand what would maximize the optical signal for low $P / P_{\mathrm{s}}$ (the main goal of a gas sensor), we have to model the adsorption. We consider a model in which the liquid-filled volume is localized into the cylindrical pore as a film adsorbed on the walls (see the inset in figure 5). As a consequence, for a sample with a given pore radius $\left(r_{\mathrm{p}}\right)$, the average thickness of the adsorbed film $(t)$ will increase with $\alpha$. From straightforward geometrical considerations, we obtain the following relationship:

$$
\alpha=\frac{V_{\text {liquid }}}{V_{\text {total }}}=\frac{\pi\left[r_{\mathrm{p}}^{2}-\left(r_{\mathrm{p}}-t\right)^{2}\right] L}{\pi r_{\mathrm{p}}^{2} L},
$$



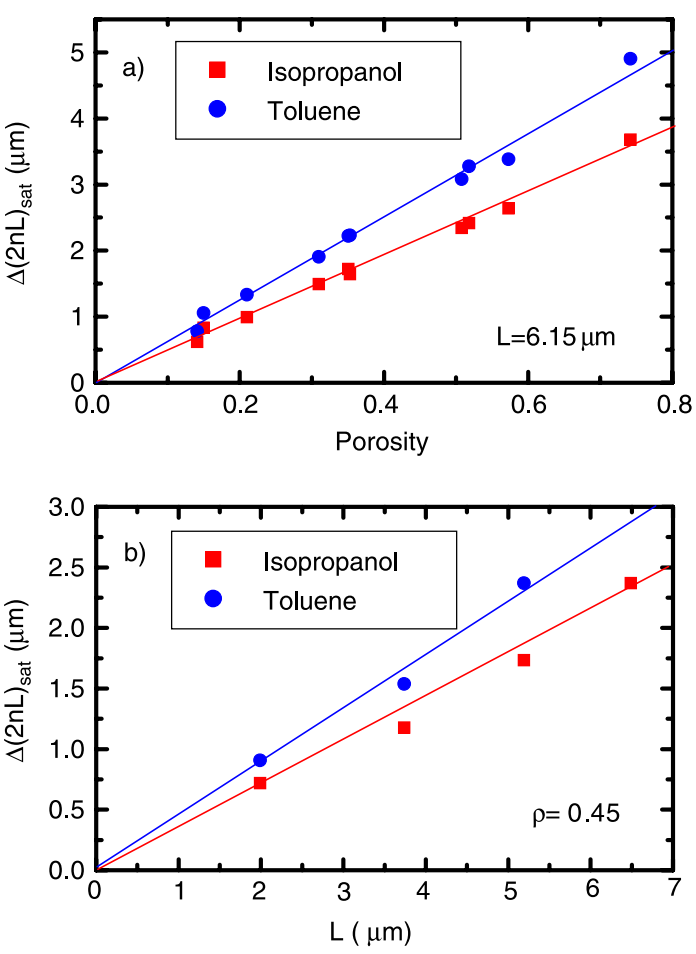

Figure 4. (a) Saturation value of the change in $2 n L$ for different analytes as a function of the porosity of the sample, for samples with the same thickness $(L=6.15 \mu \mathrm{m})$. (b) The same value as a function of the thickness of the sample, for samples with the same porosity $(\rho=0.45)$. Solid lines are a guide to the eye.

from which it is easy to see that

$$
r_{\mathrm{p}}=\frac{t}{1-\sqrt{1-\alpha}}
$$

Figure 5 shows the pore diameters of each sample as a function of $\alpha$ obtained at $P / P_{\mathrm{s}}=0.1$, for different analytes. The experimental value of $\alpha$ is obtained from the isotherms as $\alpha=\Delta(2 n L) / \Delta(2 n L)_{\text {sat }}$, following equation (3). The data fit well to equation (5), where $t$ is the only adjustable parameter, yielding $t=0.104 \pm 0.005 \mathrm{~nm}$ for isopropanol and $t=$ $0.075 \pm 0.004 \mathrm{~nm}$ for toluene. This fit is legitimate at any $P / P_{\mathrm{s}}$ below the capillary condensation, because the analyte is adsorbed as a film [5] and therefore can be described by the model (equation (5)). For every $P / P_{\mathrm{s}}$, we obtain a different $t$, consistent for all samples. This fit shows that $t$ does not depend on the pore diameter of the sample (i.e., is not affected by the confinement) and confirms the assumption $[5,22]$ that $t$ depends only on the relative pressure. According to our model, in order to optimize $\Delta(2 n L)$ at a given relative pressure, we need to maximize the volume fraction $\alpha$. Since $t$ is independent of the geometry of the pores, the pore radius has to be minimized.

In summary, for the best performance as a gas sensor we require: maximum thickness, maximum porosity and minimum pore radius. This is equivalent to maximizing the surface of the pore walls, which is where the adsorbed film is formed.

Below we discuss the capillary condensation and the associated hysteresis. The Kelvin equation, a macroscopic

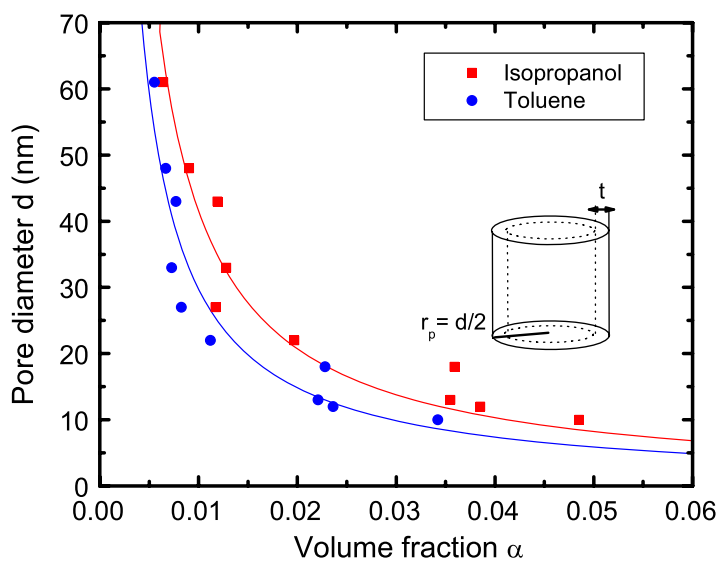

Figure 5. Pore diameter of nanoporous alumina samples as a function of the volume fraction of the pores filled with analyte (calculated from the corresponding adsorption isotherm) at $P / P_{\mathrm{s}}=0.1$. Solid lines are best fits of the model pictured in the inset (see text for details), in which an adsorbed film is formed at the pore walls. The thickness $t$ of the adsorbed film,

$t=0.104 \pm 0.005 \mathrm{~nm}$ for isopropanol and $t=0.075 \pm 0.004 \mathrm{~nm}$ for toluene, is obtained from these best fits.

thermodynamic approach which describes the meniscus (vapor-liquid interface) at equilibrium, is most frequently used to describe capillary behavior [5]:

$$
\ln \left(\frac{P}{P_{\mathrm{s}}}\right)=-\frac{2 \gamma V_{\mathrm{L}}}{R T r_{\mathrm{m}}},
$$

where $r_{\mathrm{m}}$ is the mean curvature radius of the meniscus, $R$ is the ideal gas constant and $V_{\mathrm{L}}$ and $\gamma$ are, respectively, the molar volume and surface tension of the liquid at temperature $T$. The Kelvin equation (equation (6)) is valid when (i) the pore diameters are $\geqslant 7.5-10 \mathrm{~nm}$ (otherwise, for fluids in pores with widths of a few molecular diameters, the concept of a meniscus cannot be applied) $[7,8]$ and (ii) $t$ of the adsorbed film prior to capillary condensation is negligible compared to the pore diameter (otherwise, the pore radius and meniscus radius cannot be directly related and $t$ has to be taken into account $[5,8,17])$. These two conditions are fulfilled for the samples used in this study (see figures 2 and 5, respectively).

The hysteresis in capillary phase transition has been typically explained by the Cohan model [5, 18, 22], which takes into account different shapes of the menisci during adsorption and desorption. For cylindrical pores, the evaporation in the full pore commences from the already existing hemispherical meniscus. This means that $r_{\mathrm{m}}=r_{\mathrm{p}}$ in equation (6) defines the value of $P / P_{\mathrm{s}}$ when the evaporation occurs. In contrast, for condensation in cylindrical pores without a preformed meniscus (as in case of pores open at both ends or when the closed end is of a perfect cylindrical geometry, i.e., not tapered), it is the curvature of the pore walls that determines the condensation pressure. The original Cohan model is applicable only to a wetting liquid [18], which forms a thin film of the adsorbed liquid on the pore walls. This film plays the role of a meniscus. Hence, the curvature radius of a cylindrical meniscus, $r_{\mathrm{m}}=2 r_{\mathrm{p}}$, is used in the Kelvin equation. Therefore, for the cylindrical pores open at both ends 


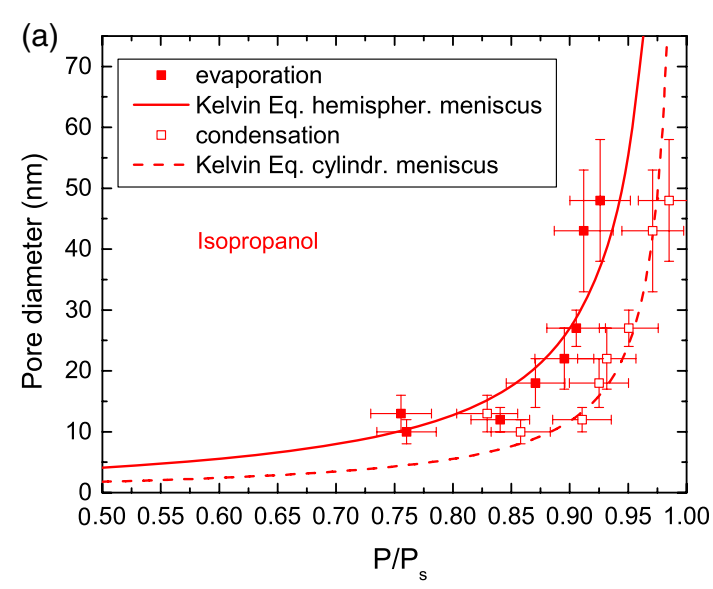

(b)

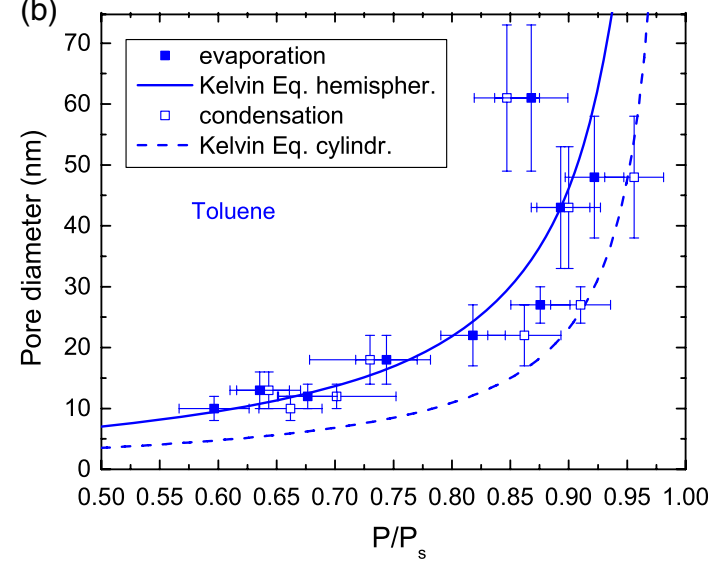

Figure 6. Pore diameter obtained from SEM plotted versus the relative pressure of (a) isopropanol and (b) toluene at which capillary condensation (open squares) and evaporation (solid squares) takes place. The value is taken as the inflection point of the adsorption and desorption isotherm, respectively, and averaged over several isotherms. Also plotted are the results obtained from the Kelvin equation for cylindrical (dashed line) and hemispherical (solid line) menisci. According to the model described in the text, these two regimes describe capillary condensation and evaporation, respectively.

or with one closed non-tapered end, condensation occurs at a $P / P_{\mathrm{S}}$ value that is higher than that for evaporation, which leads to hysteresis. The essence of this purely thermodynamical model is that, during adsorption, the adsorbed film may remain metastable beyond the equilibrium transition as given by the classical Kelvin equation (equation (6) with $r_{\mathrm{m}}=r_{\mathrm{p}}$ ) [23, 24].

The capillary condensation and evaporation transitions are shown in figure 6. The pore diameters of the samples (obtained from SEM images) are plotted as a function of the relative pressure at which condensation and evaporation occurs (taken as the inflection point of the adsorption and desorption isotherm, respectively, and averaged over multiple runs), for both isopropanol and toluene. The relation described by equation (6), using standard values of $V_{\mathrm{L}}$ and $\gamma$ for each analyte, is also plotted for the two cases of the Cohan model presented in this work (solid and dashed lines for $r_{\mathrm{m}}=r_{\mathrm{p}}$ and $r_{\mathrm{m}}=2 r_{\mathrm{p}}$, respectively). Results for isopropanol (figure 6(a)) show that condensation values are in excellent agreement with the Kelvin equation using the curvature radius of a cylindrical meniscus, whereas evaporation values are in excellent agreement with the Kelvin equation using the curvature radius of a hemispherical meniscus. This agreement is obtained without any fitting parameters, modeling of the pore structure or the adsorbed film, or other considerations. Thus, we experimentally confirm that the Cohan model quantitatively describes the condensation-evaporation hysteresis for a wetting liquid in a cylindrical pore with one flat (non-tapered) end. This confirmation is possible because the cylindrical pores in anodized alumina have a nearly ideal geometry.

The width of the transition reflects the pore size distribution (PSD) within a sample. Therefore, the PSD of a porous sample can be obtained as the derivative of its isotherm around the transition. This procedure is commonly used to characterize porous materials [5]. The conversion from relative pressure to pore diameter is usually obtained by applying the conventional Kelvin equation at equilibrium (equation (6) with $r_{\mathrm{m}}=r_{\mathrm{p}}$, i.e., hemispherical meniscus) to the experimentally determined curve. There has been much discussion in the literature as to which branch of the hysteresis loop should be used [5, 19, 22-24]. In our previous work [12], we have shown that the desorption isotherms, which occur at the $P / P_{\mathrm{s}}$ described by the Kelvin equation (equation (6)) with $r_{\mathrm{m}}=r_{\mathrm{p}}$, yield the correct PSD. To check whether adsorption curves can be used to derive the PSD, we compared the PSDs obtained from SEM images (see figure 2 for some examples) with those obtained from the derivative of the adsorption curves, using the Kelvin equation with the same parameters (equation (6) with $r_{\mathrm{m}}=r_{\mathrm{p}}$ ). These two PSDs (open squares and histogram in figure 2) are in clear disagreement. However, use of the adsorption curve together with the Kelvin equation for a cylindrical meniscus (equation (6) with $r_{\mathrm{m}}=2 r_{\mathrm{p}}$ ) recovers the correct PSD (solid circles in figure 2). This proves that the adsorption curve can also be used to obtain PSDs, provided that this transition can be described by the Cohan model.

The results for toluene are different from those for isopropanol (figure 6(b)). In this case, evaporation values are also in excellent agreement with the equilibrium state given by the hemispherical meniscus, but no clear trend is observed for adsorption values. Additionally, the desorption values for individual runs are very reproducible, whereas adsorption values have a large scattering [12]. In some cases, condensation may even occur at a pressure lower than that for evaporation. This is consistent with microscopic models [7, 23-27] which show that for independent pores, metastability should exist beyond the equilibrium transition both for adsorption (metastable vapor states) and desorption (metastable liquid states). Metastable liquid states are irrelevant for desorption, because the finite length of the pores breaks the symmetry and creates a meniscus, which can retreat on pore emptying at the equilibrium transition, as found experimentally [7, 23, 24, 26, 28]. For this reason, the pressure at which the evaporation occurs for toluene is still described by the standard Kelvin equation (equation (6)) with $r_{\mathrm{m}}=r_{\mathrm{p}}$ (figure 6(b)). On the other hand, for adsorption, the vapor condenses from nucleation in metastable states [7, 23, 28], which can be interpreted, in the macroscopic picture, as the growth of the adsorbed film [23, 24]. 
The irreproducibility of capillary condensation of toluene clearly show the relevance of fluid-solid interactions for these metastable states. These interactions influence the structure of the adsorbed layer at low relative pressures. Interactions of a polar molecule such as isopropanol [29] with the hydrophilic surface of alumina [29] stabilize the adsorbed layer [5], i.e., the wetting is almost complete and the Cohan model is applicable. In contrast, toluene is a nearly non-polar molecule [29]. A weaker interaction with the pore walls would make the metastable states more sensitive to slight fluctuations (lower energy barriers) [23], resulting in a more stochastic condensation. This weak interaction combined with a strong liquid-liquid interaction (i.e., large surface tension) in toluene [29] would favor the formation of liquid seeds, which are very sensitive to initial conditions and surface defects. In this case, the wetting is incomplete and the Cohan model is not applicable. Our observations for toluene are in agreement with Cohan's prediction that 'in the presence of incomplete wetting [...] the hysteresis should always occur', but there is 'some tendency for a drift' [18].

\section{Conclusions}

In conclusion, gas adsorption and capillary condensation of organic vapors are studied in nanoporous alumina films with non-interconnected cylindrical pores open at one end and well-controlled sizes in the $10-60 \mathrm{~nm}$ diameter range. A very sensitive optical interferometric technique is successfully used to obtain adsorption-desorption isotherms. For optimal gas sensor performance, the optical response of the film is optimized when thickness and porosity is maximized and the pore radius is minimized (i.e., the pore surface is maximum). We observe that, at low relative pressures, the average thickness of the adsorbed film is not affected by the confinement of the pores, down to $10 \mathrm{~nm}$ in diameter. We observe capillary evaporation of the liquid from the nanopores at relative pressures described by the classical Kelvin equation with a hemispherical meniscus, i.e., at the equilibrium transition, for all analytes. We quantitatively describe capillary condensation for isopropanol with the Cohan model, because wetting with alumina is complete and the cylindrical pores have a nearly ideal geometry. In this case, pore size distributions can also be obtained from the adsorption curves. However, the condensation for toluene lacks reproducibility due to incomplete wetting. This is an experimental evidence of the strong influence that van der Waals interactions have upon the metastable states that give rise to hysteresis in capillary condensation phenomenon.

\section{Acknowledgments}

The financial support of the Air Force Office of Scientific Research is gratefully acknowledged. FC acknowledges financial support from Spanish MEC and Fulbright Commission. CEC acknowledges the California Space Grant Consortion (CASGC) for a Space Grant Fellowship at the University of California, San Diego. AMR thanks the University of California, San Diego for a Graduate Assistance in Areas of National Need (GAANN) fellowship.

\section{References}

[1] Pacholski C, Sartor M, Sailor M J, Cunin F and Miskelly G M 2005 J. Am. Chem. Soc. 12711636

[2] Gao J, Gao T, Li Y Y and Sailor M J 2002 Langmuir 182229

[3] Li C-P, Roshchin I V, Batlle X, Viret M, Ott F and Schuller I K 2006 J. Appl. Phys. 100074318

[4] Gelb L D, Gubbins K E, Radhakrishnan R and Sliwinska-Bartkowiak M 1999 Rep. Prog. Phys. 621573

[5] Gregg S J and Sing K S W 1982 Adsorption, Surface Area and Porosity (London: Academic)

[6] Valiullin R, Naumov S, Galvosas P, Kärger J, Woo H-J, Porcheron F and Monson P A 2006 Nature 443965

[7] Neimark A V, Ravikovitch P I and Vishnyakov A 2000 Phys. Rev. E 62 R1493

[8] Kruk M, Jaroniec M and Sayari A 1997 Langmuir 136267

[9] Alvine K J, Shpyrko O G, Pershan P S, Shin K and Russell T P 2006 Phys. Rev. Lett. 97175503

[10] Christenson H K 1994 Phys. Rev. Lett. 731821

[11] Curry J E and Christenson H K 1996 Langmuir 125729

[12] Casanova F, Chiang C E, Li C-P, Roshchin I V, Ruminski A M, Sailor M J and Schuller I K 2008 Europhys. Lett. 8126003

[13] Masuda H and Fukuda K 1995 Science 2681466

[14] Casanova F, Chiang C E, Li C-P and Schuller I K 2007 Appl. Phys. Lett. 91243103

[15] Fisher L R and Israelachvili J N 1979 Nature 277548

[16] Kohonen M M and Christenson H K 2000 Langmuir 167285

[17] Morishige K and Tateishi M 2006 Langmuir 224165

[18] Cohan L H 1938 J. Am. Chem. Soc. 60433

[19] Sing K S W, Everett D H, Haul R A W, Moscou L, Pierotti R A, Rouquérol J and Siemieniewska T 1985 Pure Appl. Chem. 57603

[20] Bruggeman D A G 1935 Ann. Phys. 24636

[21] Xu W L, Chen H, Zheng M J, Ding G Q and Shen W Z 2006 Opt. Mater. 281160

[22] Coasne B, Grosman A, Ortega C and Simon M 2002 Phys. Rev. Lett. 88256102

[23] Ravikovitch P I, O’Domhnaill S C, Neimark A V, Schüth F and Unger K K 1995 Langmuir 114765

[24] Ball P C and Evans R 1989 Langmuir 5714

[25] Marconi U M B and Van Swol F 1989 Phys. Rev. A 394109

[26] Ravikovitch P I, Vishnyakov A, Neimark A V, Ribeiro Carrott M M L, Russo P A and Carrott P J 2006 Langmuir 22513

[27] Heffelfinger G S, van Swol F and Gubbins K E 1988 J. Chem. Phys. 895202

[28] Wallacher D, Künzner N, Kovalev D, Knorr N and Knorr K 2004 Phys. Rev. Lett. 92195704

[29] Redón R, Vázquez-Olmos A, Mata-Zamora M E, Ordóñez-Medrano A, Rivera-Torres F and Saniger J M 2005 J. Colloid Interface Sci. 287664 